\title{
Global View of Infectious Disease
}

\author{
Edwin Dias* \\ Department of Paediatrics, KVG Medical College, Rajiv Gandhi University, Mangalore-575002, Karnataka, India
}

In developing countries, the range and burden of infectious diseases are vast and of an immense variety. There are lot of new and re-emerging infectious diseases in different parts of the world. Due to globalisation there is increased travel, increased need for housing. Global warming, destruction of forests, Tsunami has only added to the problem of spread of these diseases. Infectious diseases are a leading cause of death; there have been several outbreaks of infectious diseases and have been documented since 1996. The spread of infectious diseases results as much from changes in human behaviour including lifestyles and land use patterns, increased trade and travel, and inappropriate use of antibiotic drugs as from mutations in pathogens.

Since the beginning of the year 2012 there have been outbreaks of avian influenza in Indonesia, Egypt, China, Cambodia, Vietnam, Bangladesh and in Hong Kong Special Administrative Region. This includes the outbreaks of yellow fever in Cameroon and Ghana, and meningococcal disease in African meningitis belt, with Lassa fever in Nigeria, and Undiagnosed illness in Cambodia. This also includes severe complications of Hand, Foot and Mouth Disease (HFMD) caused by EV-71 in Cambodia, Cholera outbreaks in the Democratic Republic of Congo and Sierra Leone and Ebola outbreaks in Uganda and Democratic Republic of Congo. In developed world, the spread includes Hantavirus pulmonary syndrome in Yosemite National Park, United States of America and novel corona virus infection in the United Kingdom.

The global scenario shows that twenty diseases together with tuberculosis (TB), malaria, and cholera have re-emerged or extended worldwide since 1973, often in virulent and drug-resistant forms. Thirty previously unknown disease agents have been identified since 1973, including HIV, Ebola, hepatitis C, and Nipah virus, for which no cures are available. Of the seven biggest disease killers worldwide, TB, malaria, hepatitis, and HIV/AIDS continue to increase, with HIV/AIDS and TB likely to account for the majority of deaths from infectious diseases in developing countries by 2020. Acute lower respiratory infections including pneumonia and influenza as well as diarrheal diseases and measles, appear to have peaked at high incidence levels in developing nations.

The regional trends suggest developing and former communist bloc countries will persistently experience the greatest brunt from infectious diseases as a result of malnutrition, poor sanitation, poor water quality, and insufficient health care; the developed countries also will be affected. The most vulnerable region of Sub-Saharan Africa will account for nearly half of infectious disease caused deaths worldwide. The death rates for many diseases, including HIV/AIDS and malaria, exceed those in all other regions. In certain regions of Asia and the Pacific, where multidrug resistant TB, malaria, and cholera are widespread, it is likely to observe a remarkable increase in infectious disease deaths, as a result of the spread of HIV/AIDS in South and Southeast Asia and it is likely spread to East Asia. A substantial increase in infectious disease incidence and deaths are likely in the former Soviet Union and, to a lesser extent, Eastern Europe also is likely to follow suit. Owing to an economic down turn, there has been a rapid increase in diphtheria, dysentery, cholera, and hepatitis B and C. TB has reached epidemic proportions throughout the Former Soviet Union, and HIV-infected population in Russia. Generally uneven economic development has contributed to widespread resurgence of cholera, malaria, TB, and dengue in Latin American countries. Regions of the Middle East and North Africa has substantial TB and hepatitis B and C prevalence but have limited some of the globally prevalent diseases, such as malaria and HIV/AIDS. The region has the lowest HIV infection rate among all regions. Several infectious diseases, such as HIV/AIDS, TB, and hepatitis B and C, as well as several zoonotic diseases have an impact on Western European nations, but its highly efficient health care organizations will limit the effect of these diseases.

Apart from infectious disease, rise in antibiotic resistance has caused havoc in treating disease. Rise in MRSA, MDR TB/XDR TB, New-Delhi $\beta$ lactamases in developing countries has put strain in health system. In India, the once exotic bacteria like Burkholderia pseudomallei and B. cepacia complex infections are on rise leading to mortality and morbidity. Disturbing the flora and fauna of the forest has resulted in re-emergence of Arboviruses like West Nile fever, Chikungunya and Dengue.

But emerging diseases have quadrupled in the last half-century largely because of increasing human encroachment into habitat, especially in disease "hot spots" around the globe, mostly in tropical regions. And with modern air travel and a robust market in wildlife trafficking, the potential for a serious outbreak in large population centres is enormous.

The key to forecasting and preventing the next pandemic, is to understand what they call the "protective effects" of keeping nature intact. In the Amazon, for example, one study showed that an increase in deforestation by 4 percent increased the incidence of malaria by nearly 50 percent, because mosquitoes, which transmit the disease, thrive in the right mix of sunlight and water in recently deforested areas. If actions are not taken to tackle these problems it may become a major health problem resulting in financial strain on government. Emerging Infectious Diseases (EIDs) pose a significant threat to human health, economic stability and biodiversity.

Despite this, the mechanisms underlying disease emergence are still not fully understood, and control measures rely heavily on mitigating the impact of EIDs after their emergence. Strategies to deal with EIDs proactively will require, along with continued public health investment, increased focus on identifying the driving mechanisms that highlight the emergence of each new EID and predictive modelling of how these drivers will promote or shape future EIDs' emergence potential and/or risk. These approaches are inherently multidisciplinary, and require surveillance infrastructure, logistic support, financial input and sentinel physician training during pre-pandemic Emerging infectious disease outbreaks.

*Corresponding author: Dr. Edwin Dias, Professor and HOD Paediatrics, KVG Medical College, Rajiv Gandhi University, Mangalore-575002, Karnataka, India; Fax: 701-777-2054; E-mail: dredwindias@gmail.com

Received October 15, 2012; Accepted October 16, 2012; Published October 18, 2012

Citation: Dias E (2013) Global View of Infectious Disease. J Med Microb Diagn 2: e116. doi:10.4172/2161-0703.1000e116

Copyright: (c) 2013 Dias E. This is an open-access article distributed under the terms of the Creative Commons Attribution License, which permits unrestricted use, distribution, and reproduction in any medium, provided the original author and source are credited. 